\title{
Contracepção de emergência no Brasil: desafios para a assistência farmacêutica
}

\section{Emergency contraception in Brazil: challenges for pharmaceutical care}

\author{
Elaine Reis Brandão \\ Universidade Federal do Rio de Janeiro. Instituto de Estudos em \\ Saúde Coletiva. Rio de Janeiro, RJ, Brasil. \\ E-mail: brandao®iesc.ufrj.br \\ Cristiane da Silva Cabral \\ Universidade de São Paulo. Faculdade de Saúde Pública. São \\ Paulo, SP, Brasil. \\ E-mail: cabralcsळusp.br
}

Trabalhar em um grupo de pesquisa é sempre um processo intenso de aproximação, descobertas, construção coletiva do cotidiano de trabalho, reuniões, cronogramas, tarefas, produtos, sem que nenhuma de nós tivesse dedicação exclusiva ao ofício da pesquisa. Nessa equipe, formada por colegas docentes de diferentes inserções disciplinares e/ou institucionais, por profissionais farmacêuticas que tanto nos ensinaram e nos "estranharam", por alunas que souberam estar "dentro" da equipe e "fora", ao realizarem seu próprio trabalho de pesquisa de mestrado (Bastos, 2015) e de doutorado (Paiva, 2014), sem deixarem de ser solidárias ao grupo de pesquisa, superamos dificuldades e conflitos no decorrer da investigação empreendida. ${ }^{1}$

Assim, apresentamos este dossiê, Contracepção de emergência no Brasil: desafios para a assistência farmacêutica, o qual consolida resultados da pesquisa Uma investigação socioantropológica no âmbito das farmácias/drogarias: posição de farmacêuticos e balconistas sobre a contracepção de emergência, realizada entre 2012 e 2014, no Instituto de Estudos em Saúde Coletiva, da Universidade Federal do Rio de Janeiro, com o objetivo de conhecer a visão de farmacêuticos e balconistas sobre a comercialização da contracepção de emergência em farmácias ou drogarias do país. Diversos farmacêuticos $(n=383)$ aderiram à convocação da pesquisa, respondendo espontaneamente o questionário online sobre suas representações e práticas sociais no atendimento

\section{Correspondência}

Cristiane da Silva Cabral

Universidade de São Paulo. Faculdade de Saúde Pública.

Av. Dr. Arnaldo, 715. São Paulo, SP, Brasil. CEP 01246-904.

\footnotetext{
1 A equipe de pesquisa foi composta por Elaine Reis Brandão (coordenadora), Cristiane S. Cabral, Miriam Ventura, Sabrina P. Paiva, Luiza L. Bastos, Naira de Oliveira e Iolanda Szabo. Tecemos agradecimentos especiais às instituições que nos apoiaram na divulgação do estudo entre farmacêuticos: Conselho Regional de Farmácia do Rio de Janeiro, Conselho Regional de Farmácia de São Paulo, Conselho Federal de Farmácia, Associação Brasileira de Farmácia, Rede Brasileira de Promoção de Informações e Disponibilização da Contracepção de Emergência. $\mathrm{O}$ estudo contou com apoio financeiro da Fundação de Amparo à Pesquisa do Estado do Rio de Janeiro (Faperj) e do Conselho Nacional de Desenvolvimento Científico e Tecnológico (CNPq).
} 
às consumidoras da contracepção de emergência em drogarias privadas do Brasil.

Tomamos como ponto de partida da investigação algumas premissas socioantropológicas que orientam as condutas sociais no campo da sexualidade, do gênero e da reprodução. As práticas sociais só podem ser compreendidas se inseridas no percurso biográfico dos sujeitos, articuladas a sua visão de mundo, crenças e significados (Bajos et al., 2002; Bozon, 2002; Brandão; Heilborn, 2006; Cabral, 2011; Gagnon; Simon, 1973; Heilborn et al., 2006). Além disso, subscrevemos a noção segundo a qual gênero é um importante sistema de diferenciação e hierarquização que ajuda a explicar a produção e manutenção de desigualdades sociais e de saúde (Barata, 2009; Barbieri, 1991; Heilborn, 2003). Ao se configurar como organizador da vida social, gênero é um dos determinantes majoritários das condições de saúde, adoecimento e morte. As normas de gênero estão presentes nas formas de produção e consumo de serviços de saúde, bem como nas práticas e ações dos profissionais da área. Por exemplo, sabemos que a oferta contraceptiva não é similar para homens e mulheres, tampouco o é entre mulheres de classes sociais, região, cor e etnia distintas. Propor uma leitura das práticas profissionais a partir de uma perspectiva de gênero auxilia, por um lado, a mostrar a influência insidiosa, silenciosa e naturalizante desse marcador sobre ações e condutas e, por outro, permite ressaltar a necessidade de inserir esse tipo de debate na formação dos profissionais de saúde como meio de fomentar relações interpessoais mais respeitosas e menos moralizantes e discriminatórias, no que diz respeito tanto a homens quanto a mulheres. Pode-se dizer que esse é, a um só tempo, um dos pontos de partida da investigação empreendida, mas também um dos pontos de chegada oferecido ao leitor com este dossiê.

O tema da contracepção de emergência vem sendo investigado no Brasil, na América Latina, nos EUA e Europa há algumas décadas. São exemplos: Hardy et al. (2001); Díaz et al. (2003a, 2003b); Simonds e Ellertson (2004); Martin (2004); Wynn e Trussell
(2006); Faúndes et al. (2007); Arilha, Lapa e Pisaneschi (2010); Souza (2010); Souza e Brandão (2009, 2012); Prescott (2011); Foster e Wynn (2012); Teixeira et al. (2012); e Figueiredo, Borges e Paula (2016).

No âmbito desta pesquisa, o método da contracepção de emergência foi analisado sob duas perspectivas, inscrito nas relações sociais dos sujeitos que dele necessitam e, por isso, recorrem aos serviços públicos de saúde para obtê-lo ou às farmácias/drogarias para comprá-lo. Nesta última forma de acesso ao método, as mulheres tentam garantir por si próprias, sem mediação do Estado, a obtenção do contraceptivo de emergência, que, como evidenciado pelos estudos farmacológicos, tem um tempo estabelecido de até 120 horas para surtir o efeito desejado após a relação sexual desprotegida. A segunda perspectiva de análise implica considerar a interação entre profissionais de saúde, nesse caso, farmacêuticos, e usuárias do contraceptivo de emergência, valorizando o espaço da dispensação do medicamento e da orientação clínica como potenciais aliados dos interesses das consumidoras/clientes e das boas práticas farmacêuticas. ${ }^{2}$

Nesse sentido, o dossiê traz para o centro do debate público sobre contracepção de emergência a categoria profissional dos farmacêuticos, até então ausente dessa discussão no Brasil. Como grande parte da população recorre às drogarias para adquirir contracepção de emergência e os dados do IMS Health, um instituto de pesquisa voltado ao marketing farmacêutico, indicam crescente aumento no faturamento dos laboratórios farmacêuticos com as vendas das marcas disponíveis no mercado brasileiro nos últimos anos (Figueiredo, 2016), decidimos ouvir tal segmento profissional, conhecer o que pensa a respeito desse tipo de medicamento e de seus consumidores.

A literatura científica produzida sobre o tema no Brasil contempla prioritariamente outros profissionais de saúde (enfermeiros, médicos), gestores, usuárias e estudantes universitários e da educação básica, não havendo inclusão do farmacêutico no debate em curso sobre o acesso em tempo oportuno ao método contraceptivo.

\footnotetext{
2 Cf. Resoluções do Conselho Federal de Farmácia n ${ }^{0} 585$, de 29 de agosto de 2013, que regulamenta as atribuições clínicas do farmacêutico e dá outras providências, e $\mathrm{n}^{0} 586$, de 29 de agosto de 2013 , que regula a prescrição farmacêutica e dá outras providências.
} 
Ao contrário, a revisão da literatura científica internacional sobre as políticas de provisão da contracepção de emergência, notadamente por meio das farmácias (Paiva; Brandão, 2012), nos apontou duas perspectivas analíticas: a disponibilidade e as barreiras à contracepção de emergência nas farmácias, situando a posição dos farmacêuticos em relação ao método; e a posição das mulheres consumidoras a respeito do contraceptivo e de seus locais de provisão. Tais estudos evidenciaram que a adoção de políticas públicas favoráveis à dispensação da contracepção de emergência pelas farmácias contribui, de modo geral, para uma avaliação positiva de farmacêuticos e usuários, principalmente devido à possibilidade de dispensar o medicamento de forma mais ágil. 0 tempo é um elemento central no acesso a esse método.

Os países que adotaram tal política discutem a necessidade de aconselhamento em saúde sexual e reprodutiva para os consumidores no âmbito da própria farmácia. Entretanto, no Brasil, o medicamento é vendido sem a mulher receber necessariamente orientação do farmacêutico, pois não há política pública na área do planejamento reprodutivo que inclua a provisão dos contraceptivos hormonais pelas farmácias/drogarias ou discuta sua venda livre (OTC - over the counter).

A pesquisa realizada entre 2012 e 2014, à qual já aludimos, produziu e divulgou no início do ano de 2016 o site www.piluladeemergencia.com.br com material didático e educativo voltado à atualização dos profissionais do comércio farmacêutico, por meio da campanha "Pílula de emergência: ética no atendimento e respeito à consumidora!”. Nosso objetivo com tal iniciativa foi devolver alguns resultados da investigação e disponibilizar, aos interessados, referências e fontes de informação sobre o tema para esclarecimento de dúvidas.

O dossiê reúne quatro artigos que discutem de modos distintos o assunto investigado. 0 primeiro, intitulado "Articulações entre contracepção, sexualidade e relações de gênero", de autoria de Cristiane S. Cabral (2017), da Faculdade de Saúde Pública da Universidade de São Paulo, aborda a questão da cultura contraceptiva no Brasil, apresentando um panorama atualizado dos imensos desafios postos àqueles que trabalham com o tema da reprodução na área da saúde, em suas articulações com sexualidade e relações de gênero. A partir do agudo conhecimento das trajetórias sexuais, contraceptivas e reprodutivas das mulheres no país, tecidas mediante determinações estruturais de classe social e gênero e capacidades subjetivas de agência e negociação, podemos refletir sobre dificuldades e desafios na atenção à saúde das mulheres. A argumentação de que há um aprendizado do manejo contraceptivo, que é profundamente marcado por outras dimensões, como fatores macroestruturais, relacionais e individuais, procura tornar mais complexo o debate sobre gestações imprevistas e se antepor à uma concepção racionalista ou de responsabilização individual dos eventos reprodutivos. As formas de disponibilização, acesso e recurso à contracepção de emergência precisam ser compreendidas a partir desse enquadramento multifatorial, do qual compartilham usuárias e profissionais de saúde.

No segundo artigo, o leitor encontrará os resultados da consulta aos farmacêuticos sobre o cotidiano de atuação profissional em farmácias ou drogarias do país. Apresentando o perfil dos entrevistados e algumas dificuldades que estes encontram para uma prática profissional voltada aos interesses da população que os procura, as farmacêuticas Naira Vidal de Oliveira, Iolanda Szabo e Luiza Lena Bastos (2017), da Faculdade de Farmácia e do Instituto de Estudos em Saúde Coletiva da Universidade Federal do Rio de Janeiro, em diálogo com Sabrina Pereira Paiva, problematizam alguns importantes aspectos da prática farmacêutica, inclusive suas percepções/representações enquanto profissionais de saúde.

No terceiro artigo, Elaine Reis Brandão (2017), do Instituto de Estudos em Saúde Coletiva da Universidade Federal do Rio de Janeiro, discute o atendimento farmacêutico às consumidoras da contracepção de emergência, complementando os resultados da pesquisa já divulgados (Brandão et al., 2016a, 2016b, 2017). Em geral, nota-se tanto entre farmacêuticos, quanto entre balconistas de farmácias e drogarias a percepção de um suposto uso abusivo da contracepção de emergência por jovens mulheres, a princípio não verificado em outros estudos que problematizam a utilização 
desse tipo de medicamento no país (Chofakian, 2017; Chofakian; Borges; Santos, 2016). A reificação da concepção, muito encontrada no senso comum sobre tal modalidade de contraceptivo, ou seja, a designação da contracepção de emergência como "bomba hormonal" persiste como categoria estruturante entre os trabalhadores de farmácias. Novas investigações serão bem-vindas para problematizar as dimensões desse consumo e o lugar da contracepção de emergência dentre as alternativas contraceptivas disponíveis às mulheres do Brasil. No bojo dos processos de medicalização e de farmaceuticalização da vida social, acredita-se que os farmacêuticos que atuam em estabelecimentos dessa natureza no país possam ter um lugar privilegiado no cuidado à saúde da população, intervindo de maneira reflexiva e crítica na orientação clínica durante a dispensação dos contraceptivos. A questão do uso racional de medicamentos é abordada mediante as tensões derivadas de práticas de automedicação em contextos culturais peculiares, que respondem a lógicas sociais específicas/particulares.

No último artigo, Rogerio Lopes Azize (2017), do Instituto de Medicina Social da Universidade do Estado do Rio de Janeiro, comenta criticamente o material aqui reunido, aceitando o convite das editoras da revista, que solicitaram ao pesquisador um olhar distanciado e reflexivo sobre os resultados trazidos. Com sua expertise na área da antropologia dos medicamentos, muito nos honra receber tais considerações, com as quais certamente aprenderemos novas lições.

Os desafios da afirmação da autonomia sexual e reprodutiva por parte das mulheres é o motor que nos move. Esperamos que os leitores apreciem e possam debater algumas destas ideias em suas práticas de saúde cotidianas.

\section{Referências}

ARILHA, M.; LAPA, T. S.; PISANESCHI, T. C. (Org.). Contracepção de emergência no Brasil e América Latina: dinâmicas políticas e direitos sexuais e reprodutivos. São Paulo: Oficina Editorial, 2010.

AZIZE, R. L. Contracepções diversas: uma leitura crítica do dossiê "Contracepção de emergência no Brasil: desafios para a assistência farmacêutica”. Saúde e Sociedade, São Paulo, v. 26, n. 4, p. 1156-1162, 2017.

BAJOS, N. et al. De la contraception à l'avortement: sociologie des grossesses non prévues. Paris:

Inserm, 2002. $345 \mathrm{p}$.

BARATA, R. B. Como e por que as desigualdades sociais fazem mal à saúde. Rio de Janeiro:

Fiocruz, 2009. 120 p.

BARBIERI, T. Sobre la categoría de género: una introducción teórico metodológica. In: AZERÊDO, S.; STOLCKE, V. (Org.). Direitos reprodutivos. São Paulo: Prodir: Fundação Carlos Chagas, 1991. p. 25-46.

BASTOS, L. L. O Consórcio Internacional sobre Contracepção de Emergência: um estudo dos argumentos para difusão dos contraceptivos de emergência em países em desenvolvimento. 2015. Dissertação (Mestrado em Saúde Coletiva) - Instituto de Estudos em Saúde Coletiva, Universidade Federal do Rio de Janeiro, Rio de Janeiro, 2015.

BOZON, M. Sociologie de la sexualité. Paris: Nathan, 2002.

BRANDÃO, E. R. O atendimento farmacêutico às consumidoras de contracepção de emergência. Saúde e Sociedade, São Paulo, v. 26, n. 4, p. 1142-1155, 2017.

BRANDÃO, E. R.; HEILBORN, M. L. Sexualidade e gravidez na adolescência entre jovens de camadas médias do Rio de Janeiro, Brasil. Cadernos de Saúde Pública, Rio de Janeiro, v. 22, n. 7, p. 14211430, 2006.

BRANDÃO, E. R. et al. O olhar do farmacêutico sobre a contracepção de emergência. In: FIGUEIREDO, R.; BORGES, A. L. V.; PAULA, S. H. B. (Org.). Panorama da contracepção de emergência no Brasil. São Paulo: Instituto de Saúde, 2016a. p. 167-192.

BRANDÃO, E. R. et al. "Bomba hormonal”: os riscos da contracepção de emergência na perspectiva dos balconistas de farmácias no Rio de Janeiro, Brasil. Cadernos de Saúde Pública, Rio de Janeiro, v. 32, n. 9, p. 2-11, 2016b. 
BRANDÃO, E. R. et al. Os perigos subsumidos na contracepção de emergência: moralidades e saberes em jogo. Horizontes Antropológicos, Porto Alegre, v. 23, n. 47, p. 131-161, 2017.

CABRAL, C. S. Práticas contraceptivas e gestão da heterossexualidade: agência individual, contextos relacionais e gênero. 2011. Tese (Doutorado em Saúde Coletiva) - Instituto de Medicina Social, Universidade do Estado do Rio de Janeiro, Rio de Janeiro, 2011.

CABRAL, C. S. Articulações entre contracepção, sexualidade e relações de gênero. Saúde e Sociedade, n. 26, v. 4, p. 1113-1124, 2017.

CHOFAKIAN, C. B. N. Contraceptive discontinuation and its relation to emergency contraception use among undergraduate women. 2017. Tese (Doutorado em Ciências) Escola de Enfermagem, Universidade de São Paulo, São Paulo, 2017.

CHOFAKIAN, C. B. N.; BORGES, A. L. V.; SANTOS, O. A. Conhecimento e uso de anticoncepção de emergência entre adolescentes. In: FIGUEIREDO, R.; BORGES, A. L. V.; PAULA, S. H. B. (Org.). Panorama da contracepção de emergência no Brasil. São Paulo: Instituto de Saúde, 2016. p. 125-143.

DÍAZ, S. et al. Acceptability of emergency contraception in Brazil, Chile, and Mexico: 1: perceptions of emergency oral contraceptives. Cadernos de Saúde Pública, Rio de Janeiro, v. 19, n. 5, p. 1507-1517, 2003 .

DÍAZ, S. et al. Acceptability of emergency contraception in Brazil, Chile, and Mexico: 2: facilitating factors versus obstacles. Cadernos de Saúde Pública, Rio de Janeiro, v. 19, n. 6, p. 17291737, 2003b.

FAÚNDES, A. et al. Emergency contraception under attack in Latin America: response of the medical establishment and civil society. Reproductive Health Matters, London, v. 15, n. 29, p. 130-138, 2007.

FIGUEIREDO, R. Panorama da distribuição gratuita, venda e uso da contracepção de emergência no Brasil. In: FIGUEIREDO, R.; BORGES, A. L. V.; PAULA, S. H. B. (Org.). Panorama da contracepção de emergência no Brasil. São Paulo: Instituto de Saúde, 2016. p. 83-102.

FIGUEIREDO, R.; BORGES, A. L. V.; PAULA, S. H. B. (Org.). Panorama da contracepção de emergência no Brasil. São Paulo: Instituto de Saúde, 2016.

FOSTER, A.; WYNN, L. L. (Ed.). Emergency contraception: the story of a global reproductive health technology. New York: Palgrave Macmillan, 2012.

GAGNON, J.; SIMON, W. Sexual conduct: the social sources of human sexuality. Chicago: Aldine, 1973. p. 316.

HARDY, E. et al. Anticoncepção de emergência no Brasil: facilitadores e barreiras. Cadernos de Saúde Pública, Rio de Janeiro, v. 4, n. 17, p. 10311035, 2001.

HEILBORN, M. L. Articulando gênero, sexo e sexualidade: diferenças na saúde. In: GOLDENBERG, P.; MARSIGLIA, R. M. G.; GOMES, M. H. A. (Org.). O clássico e o novo: tendências, objetos e abordagens em ciências sociais e saúde. Rio de Janeiro: Fiocruz, 2003. p. 197-208.

HEILBORN, M. L. et al. (Org.). O aprendizado da sexualidade: reprodução e trajetórias sociais de jovens brasileiros. Rio de Janeiro: Garamond: Fiocruz, 2006.

MARTIN, A. La anticoncepción de emergencia en América Latina y el Caribe. Revista Panamericana de Salud Pública, Washington, DC, v. 16, n. 6, p. 424-31, 2004.

OLIVEIRA, N. V. et al. Atuação profissional dos farmacêuticos no Brasil: perfil sociodemográfico e dinâmica de trabalho em farmácias e drogarias privadas. Saúde e Sociedade, São Paulo, v. 26, n. 4, p. 1125-1141, 2017.

PAIVA, S. P. Silêncio, não dito e vergonha no balcão da drogaria: estudo etnográfico sobre a comercialização da contracepção de emergência no Rio de Janeiro/RJ. 2014. Tese (Doutorado em Saúde Coletiva) - Instituto de Estudos em Saúde Coletiva, Universidade Federal do Rio de Janeiro, Rio de Janeiro, 2014. 
PAIVA, S. P.; BRANDÃO, E. R. Contracepção de emergência no contexto das farmácias: revisão crítica de literatura. Physis: Revista de Saúde Coletiva, Rio de Janeiro, v. 22, n. 1, p. 17-34, 2012.

PRESCOTT, H. M. The morning after: a history of emergency contraception in the United States. New Jersey: Rutgers University, 2011.

SIMONDS, W.; ELLERTSON, C. Emergency contraception and morality: reflections of health care workers and clients. Social Science \& Medicine, Oxford, v. 58, n. 7, p. 1285-1297, 2004.

SOUZA, R. A. O debate social sobre a anticoncepção de emergência no Brasil (2005-2008). 2010. Dissertação (Mestrado Saúde Coletiva) - Instituto de Estudos em Saúde Coletiva, Universidade Federal do Rio de Janeiro, Rio de Janeiro, 2010.

SOUZA, R. A.; BRANDÃO, E. R. Marcos normativos da anticoncepção de emergência e as dificuldades de sua institucionalização nos serviços públicos de saúde. Physis: Revista de Saúde Coletiva, Rio de Janeiro, v. 14, n. 19, p. 1067-86, 2009.

SOUZA, R. A.; BRANDÃO, E. R. À sombra do aborto: o debate social sobre a anticoncepção de emergência na mídia impressa brasileira (2005-2009). Interface: Comunicação, Saúde e Educação, Botucatu, v. 16, n. 4o, p. 161-176, 2012.

TEIXEIRA, M. et al. Representations and uses of emergency contraception in West Africa: a social anthropological reading of a northern medicinal product. Social Science \& Medicine, Oxford, v. 75, n. 1, p. 148-155, 2012.

WYNN, L. L.; TRUSSELL, J. The social life of emergency contraception in the United States: disciplining pharmaceutical use, disciplining sexuality, and constructing zygotic bodies. Medical Anthropology Quarterly, Washington, DC, v. 20, n. 3, p. 297-320, 2006.

\section{Contribuição das autoras}

Ambas as autoras participaram de todas as fases de elaboração do manuscrito.

Recebido: 22/09/2017

Aprovado: $24 / 10 / 2017$ 